\title{
Damping Applications of Ferrofluids: A Review
}

\author{
Chuan Huang ${ }^{1}$, Jie Yao ${ }^{1}$, Tianqi Zhang ${ }^{1}$, Yibiao Chen ${ }^{2}$, Huawei Jiang ${ }^{1}$, and Decai Li ${ }^{1,3 *}$ \\ ${ }^{1}$ School of Mechanical, Electronic and Control Engineering, Beijing Jiaotong University, Beijing 100044, China \\ ${ }^{2}$ School of Mechanical Engineering, University of Science and Technology Beijing, Beijing 100083, China \\ ${ }^{3}$ State Key Laboratory of Tribology, Tsinghua University, Beijing 100084, China
}

(Received 8 November 2016, Received in final form 10 January 2017, Accepted 11 January 2017)

\begin{abstract}
Ferrofluids are a special category of smart nanomaterials which shows normal liquid behavior coupled with superparamagnetic properties. One of the earliest and most prospective applications of ferrofluids is in damping, which has prominent advantages compared with conventional damping devices: simplicity, flexibility and reliability. This paper presents the basic principles that play a major role in the design of ferrofluid damping devices. The characteristics of typical ferrofluid damping devices including dampers, vibration isolators, and dynamic vibration absorbers are compared and summarized, and then recent progress of vibration energy harvesters based on ferrofluid is briefly described. Additionally, we proposed a novel ferrofluid dynamic vibration absorber in this paper, and its damping efficiency was verified with experiments. In the end, the critical problems and research directions of the ferrofluid damping technology in the future are raised.
\end{abstract}

Keywords : ferrofluid, damping applications, damper, vibration isolator, dynamic vibration absorber, vibration energy harvester

\section{Introduction}

Ferrofluids, also known as magnetic fluids, are a special category of smart nanomaterials [1]. The model of a ferrofluid is a three-component material with one homogeneous phase. A ferrofluid consists typically of a suspension of monodomain ferromagnetic particles such as magnetite in a nonmagnetic carrier fluid. A surfactant covering the particles prevents particle-to-particle agglomeration, and Brownian motion avoids particle sedimentation in gravitational or magnetic fields [2]. The fluid exhibits both fluidity and superparamagnetism, and thus its flow and properties can be controlled with the help of magnetic fields [1].

Originally as a way to control liquids in the microgravity environment of space, the first synthesis of stable ferrofluid was developed by Papell [3] of the National Aeronautics and Space Administration (NASA) in the early 1960's. In particular, ferrofluid should not be confused with the magnetorheological fluid (MR fluid) which

CThe Korean Magnetics Society. All rights reserved.

*Corresponding author: Tel: +86-10-51684006

Fax: +86-10-51685265, e-mail: dcli@bjtu.edu.cn was first introduced by Rabinow [4] in 1948. On the one hand, both of them are magnetic field-responsive fluids, and are called magnetic fluids in some cases [5]. On the other hand, consisting of micron size particles, MR fluid possesses the unusual performance to encounter rapid (within a few milliseconds), nearly completely reversible, and great changes in its intensity under the effect of an external magnetic field, in other words, from a freeflowing state to a solid like state [6]. MR fluid based devices, especially MR fluid dampers, are commonly used in civil engineering applications, medical applications, and automotive applications [7].

However, the relatively "softer" ferrofluid, which could maintain its liquid properties under the magnetic field, has played a vital role in solving complex engineering problems. The salient features of ferrofluids have attracted great interest in the research of a wide range of ferrofluid based devices such as seals, sensors, bearings, micro pumps and damping applications. Among them, damping applications are the earliest and most prospective applications of ferrofluids.

Ferrofluid damping applications have the advantages of long life, no leakage, more compact structure, less energy consuming, etc., when compared to conventional fluid 
damping devices, because of the main characteristics of ferrofluids, for example, their response to applied magnetic fields and levitation of magnetic and nonmagnetic objects [8]. It is noteworthy that, the prevention of leakage can be achieved in space, since the ferrofluid could be precisely captured and positioned by an external magnetic field.

By virtue of the viscous characteristics, ferrofluid viscous dampers became the earliest damping devices of ferrofluids which have attracted worldwide attention. It was first reported in 1967 by Goddard Space Flight Center of NASA, which was designed to reduce the oscillations of a Radio Astronomy Explorer (RAE) type of satellite [9]. Soon after, Leo and Rudolph [10] filed an application for a patent on a viscous damper using ferrofluid. From 1970's to 1990's, Raj et al. [11-14] discussed commercial applications of ferrofluid damping, and other researchers [15-20] introduced ferrofluid damping applications as an important part in their papers. It is worth noting that Raj and Moskowitz [21] have made an excellent review on damping applications of ferrofluids in 1980, including rotary viscous inertia damper, linear damper, damper/seal assembly, and dashpot. In 2014, Torres-Díaz and Rinaldi [22] made an outstanding review of recent advances in established and emerging applications of ferrofluids, including applications in optics, sensors, actuators, seals, lubrication, etc., however, little about damping applications of ferrofluids was mentioned.

In recent years, ferrofluids have shown their possibilities in many emerging applications, such as in optical fields [23], biomedical applications [24], and microfluidic systems [25]. While in this study, we focus on the traditional and promising applications of ferrofluids, namely damping applications.

In order to mark the significance of research and development of ferrofluid damping applications over half a century, a comprehensive review is needed. This can serve as a useful supplement to existing literature which was made a few decades ago or recent reviews with respect to ferrofluid applications but with little focus on damping.

This review begins with an introduction of some essential properties and theories related to damping applications of ferrofluids. Then typical damping applications are discussed according to the vibration control methods: dampers, vibration isolators, and dynamic vibration absorbers. Following this, the recent progress of vibration energy harvesters based on ferrofluid is briefly provided. In addition, we proposed a novel ferrofluid dynamic vibration absorber in this paper, and its damping efficiency was verified with experiments. The critical problems and research directions of the ferrofluid damping technology in the future are described in the last section.

\section{Basic Principles of Ferrofluid Damping Applications}

Ferrofluid damping applications mainly lie in the special characters of ferrofluids. Basic principles of ferrofluids are given as following.

\subsection{Ferrohydrodynamic equations}

The ferrohydrodynamic Navier-Stokes equation is obtained as [26]:

$$
\rho\left(\frac{\partial \boldsymbol{V}}{\partial t}+\boldsymbol{V} \cdot \nabla \boldsymbol{V}\right)=-\nabla p^{*}+\mu_{0} \boldsymbol{M} \cdot \nabla \boldsymbol{H}+\eta \nabla^{2} \boldsymbol{V}+\rho \boldsymbol{g}
$$

Where $\rho$ is the density of ferrofluid, $\boldsymbol{V}$ is the velocity of ferrofluid, $\eta$ is the viscosity of ferrofluid, $\boldsymbol{g}$ is the local acceleration due to gravity, and $p^{*}$ is the composite pressure, which is given as follows [26]:

$$
p^{*}=p(\rho, T)+p_{\mathrm{s}}+p_{\mathrm{m}}
$$

Where $p_{\mathrm{s}}$ is the magnetostrictive pressure, and $p_{\mathrm{m}}$ is the fluid-magnetic pressure.

\subsection{Passive levitation of a nonmagnetic object}

Figure 1 is a container filled with a ferrofluid placed between the like poles of two bar magnets of equal strength, in which the magnetic energy is highest at the center of the fluid and decreases symmetrically outward. Contrarily, the pressure is lowest at the center and increases with distance since the sum of the magnetic energy and the pressure must be constant everywhere. So when a nonmagnetic object is positioned in the container, a striking technological force is generated on the nonmagnetic

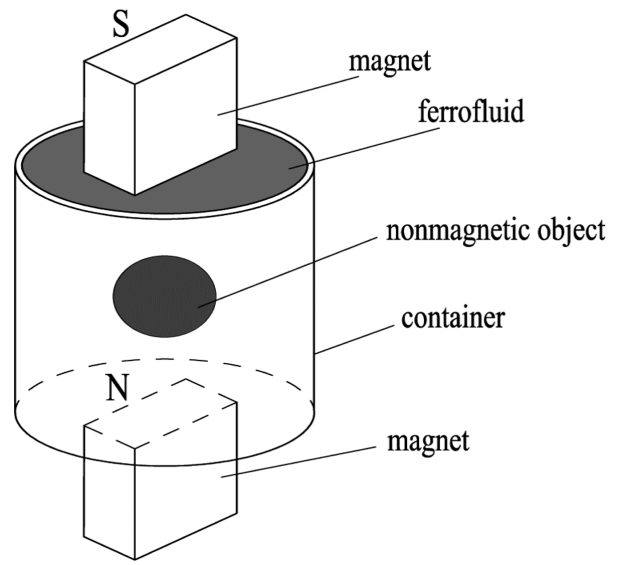

Fig. 1. Passive levitation of a nonmagnetic object in a ferrofluid. 


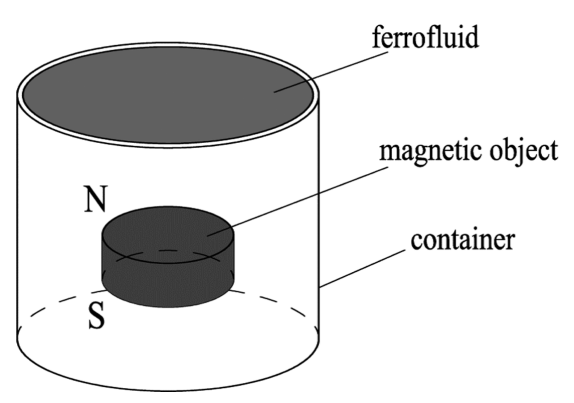

Fig. 2. Self-levitation of a magnetic object in a ferrofluid.

object, thus the nonmagnetic object moves to the center and remains there in equilibrium [26]. This phenomenon is denoted as passive levitation of a nonmagnetic object [26], or buoyant levitation of the first kind [27].

The magnetic buoyancy force $\boldsymbol{F}_{\mathrm{m}}$ exerted on such a nonmagnetic body of volume $V$ is given by [26]:

$$
\boldsymbol{F}_{\mathrm{m}}=\mu_{0} \boldsymbol{M} \cdot \nabla \boldsymbol{H}
$$

Where $\boldsymbol{M}$ is the magnetization of the ferrofluid, $\nabla \boldsymbol{H}$ is the gradient of the magnitude of the magnetic field, and $\mu_{0}$ is the permeability constant.

\subsection{Self-levitation of a magnetic object}

Figure 2 illustrates a magnetic unit immersed in a container full of ferrofluid will tend to move to the interior of the fluid space where the magnetic field is strongest near the magnetic object, even its density is greater than that of ferrofluid $[26,28]$. This phenomenon is denoted as selflevitation of a magnetic object [26], or buoyant levitation of the second kind [27].

\section{Typical Damping Applications of Ferrofluids}

Typical damping applications of ferrofluids are reviewed in this section under the classifications of dampers, vibration isolators, and dynamic vibration absorbers, according to the vibration control methods. Moreover, applications of ferrofluids in vibration energy harvesters, which is an emerging area of importance, are elaborated briefly. Most of these devices presented in the literature are summarized in tables.

\subsection{Ferrofluid Dampers}

Among the earliest applications of ferrofluids, ferrofluid dampers have obvious advantages over conventional dampers for their simplicity, reliability, and lightweight which originate from two characteristics of ferrofluids. First, the ferrofluid must be susceptible to be captured by a magnetic field. Second, the ferrofluid must have suffi-

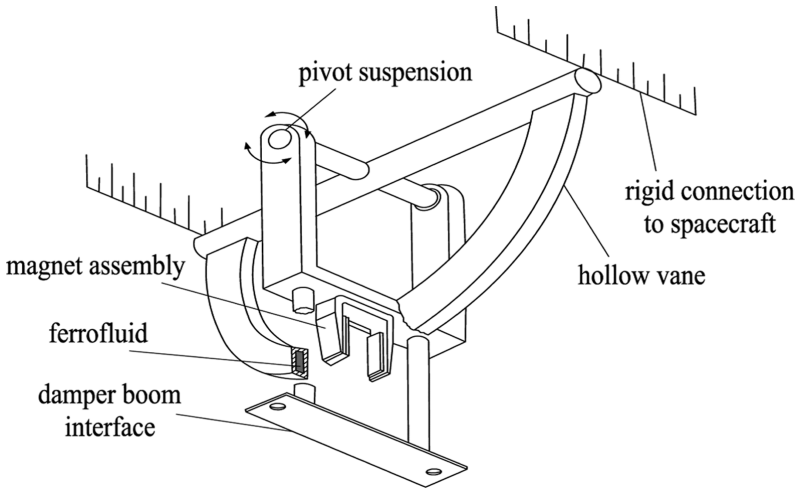

Fig. 3. A ferrofluid viscous damper for a RAE type of satellite.

cient viscosity for its use either as a damper or as a coupling device. Because of the unique characteristic of its response to the magnetic field, a ferrofluid damper absorbs the motion energy by a shearing effect which produces a torque that opposes the unwanted oscillatory motion. It was originally developed for space technology, and was soon applied in other areas.

Figure 3 shows a ferrofluid viscous damper for a RAE type of satellite [9]. It made use of an energy dissipation damping mechanism to couple the damper boom and the central body of the satellite together. When the damper boom has an angular motion with respect to the satellite central body, causing velocity sensitive damping forces applied to the system, then energy dissipation occurs. The feasibility of the concept was established, and a model was developed and fabricated to demonstrate the principle of operation. Alpha methyl naphthalene was chosen as the carrier of the ferrofluid, owing to the characteristics requirement of the damping fluid, including thermal-viscosity characteristics within necessary limits. The damper was quite light and its total weight could be about 0.38 $\mathrm{kg}$.

Figure 4 is another ferrofluid viscous damper [10] with the primary advantage of simplicity which based on the

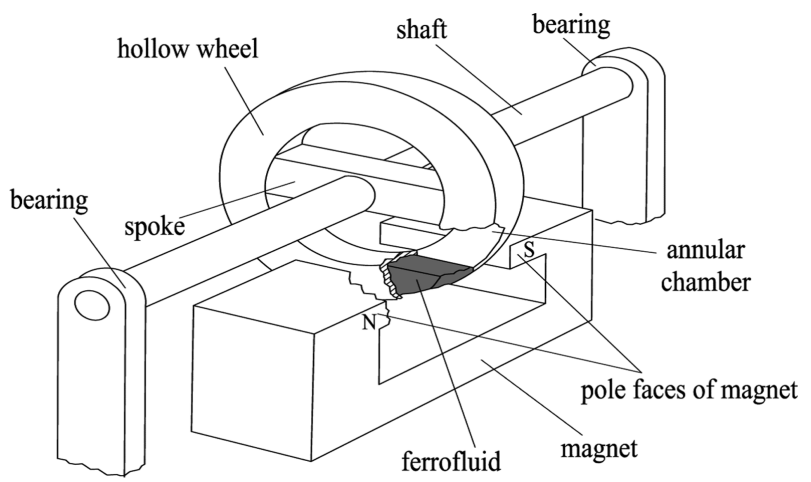

Fig. 4. A ferrofluid viscous damper with one wheel. 


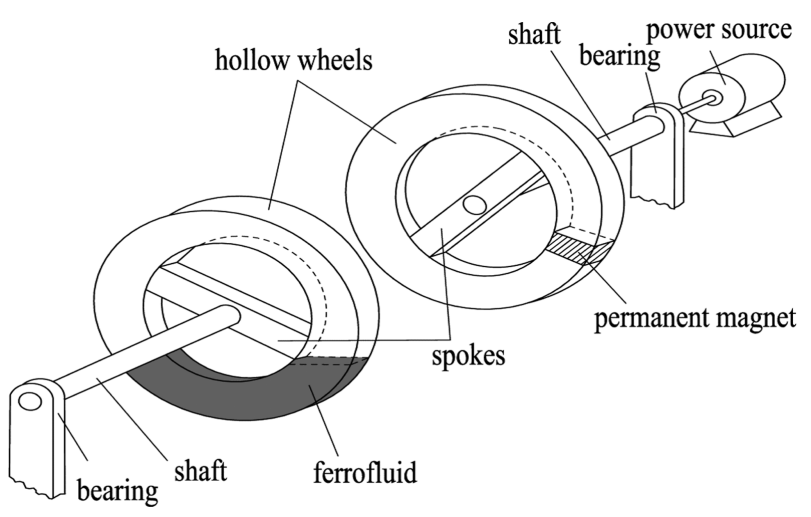

Fig. 5. A ferrofluid viscous damper with two wheels.

same principles as the device in Fig. 3. A hollow wheel made of nonmagnetic materials is arranged between two faces of a magnet with a concave structure, and ferrofluid is enclosed in the annular chamber formed by the hollow wheel. Relative velocity between the wheel and magnet caused by oscillation of the wheel will bring about energy dissipation, due to the viscous shear forces generated in the ferrofluid, which in fact are proportional to the relative velocity. This kind of viscous damper can be applied in dynamic systems where the magnet and wheel are attached to two elements, respectively. Furthermore, Leo and Rudolph [10] presented another embodiment based on coupling mechanism, which has two wheels, equipped with ferrofluid and magnets, respectively, as can be seen in Fig. 5. Both configurations have many deformation structures.

Based on the magnetorheologic effects of ferrofluid, researchers from Romania [29] put forward a ferrofluid brake, and the schematic diagram is shown in Fig. 6. A ferromagnetic disk is placed in an enclosed carcass filled with ferrofluid. Eight electromagnets which could generate a magnetic field transversal on the flow direction of ferro-

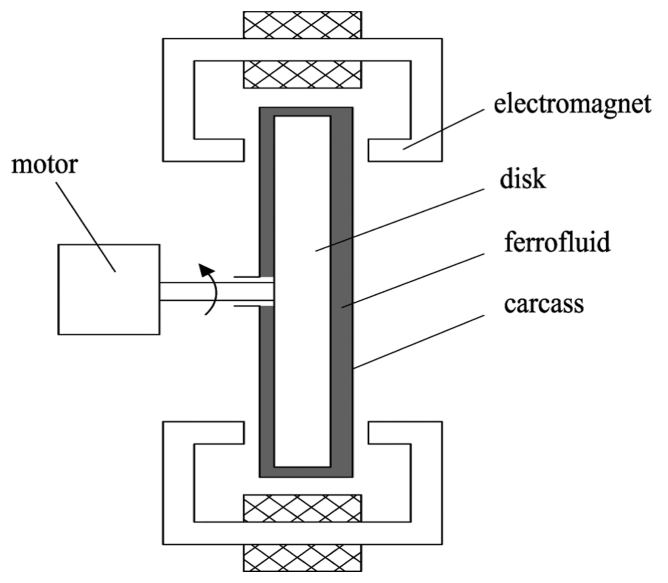

Fig. 6. A ferrofluid brake.

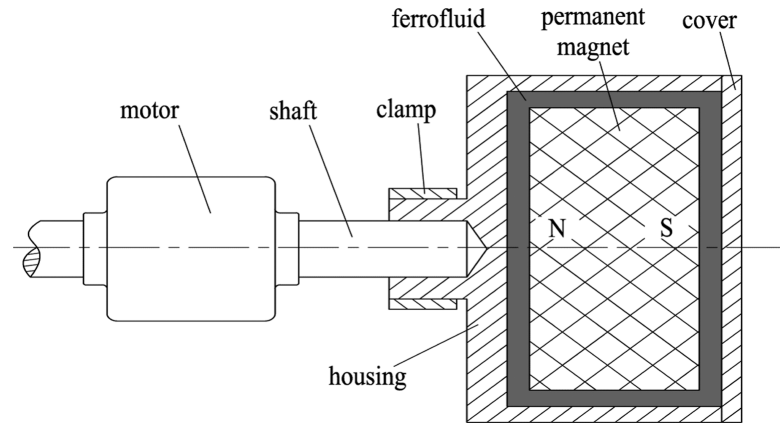

Fig. 7. A ferrofluid inertia damper based on levitation of a magnetic object.

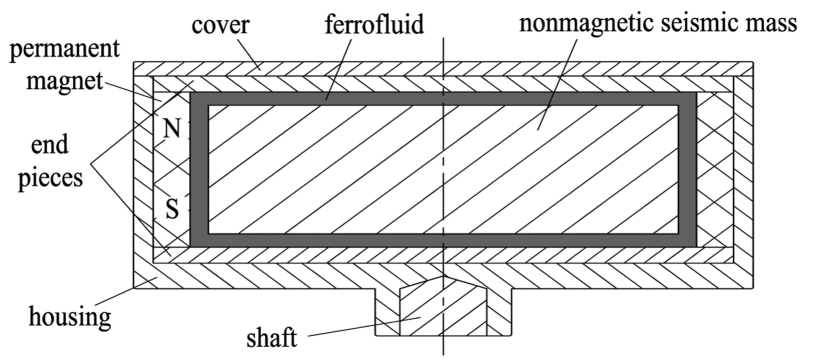

Fig. 8. A ferrofluid inertia damper based on levitation of a nonmagnetic object.

fluid are disposed outside the carcass. By adjusting the current intensity supplied to the electromagnets, the strength of the magnetic field can be changed. The influence of the magnetic field versus the power dissipated by a disk brake under various speed conditions was studied.

All ferrofluid dampers described above have no direct contact between ferrofluid and magnets. In accordance to the unique phenomenon of ferrofluid levitation, Moskowitz et al. [30] proposed another type of ferrofluid damper, exactly called a ferrofluid inertia damper. Figure 7 is a schematic cross-sectional illustration of an inertia damper, in which the seismic mass containing a permanent magnet is levitated in the chamber. Thus the bearings are not needed to support the seismic mass. Viscous shear forces originating from ferrofluid disposed between the wall surface of the chamber and the seismic mass leads to energy consumption of dynamic system. This type of viscous inertia dampers is commonly employed with stepper motors and similar devices to absorb the rotation energy in the process of stopping. Due to the levitation effect of ferrofluid on magnetic or nonmagnetic objects in certain circumstances, the seismic mass can also be made of nonmagnetic material, while a ring magnet was needed in the chamber (see Fig. 8). Compared to the self-levitation of a magnetic object in a ferrofluid, actually, the suspension of a nonmagnetic mass is not easy, so special structural optimization design is required. 




Fig. 9. A rotary viscous damper using ferrofluid.

Based on the dampers proposed by Moskowitz et al. [30], there produced some improved structures, one of which can be seen in Fig. 9 [31]. Compared with the structure in Fig. 7, it is not necessary to get the seismic mass levitated. A low reluctance magnetic path is formed in its magnetically permeable housing, concentrating the magnetic field in the region between the rotor and the inner walls of the housing, and the ferrofluid is attracted to the region with the maximum magnetic field intensity. So the damping ferrofluid is held away from the apertures and within the housing. Accordingly, mechanical fluid seals are not required.

In actual working environment, temperature fluctuations are real problems for these devices discussed above. To eliminate the influence caused by thermal fluctuations, scholars from Japan [32] proposed an improved configuration (see Fig. 10). There is a difference in coefficient of thermal expansion between the materials of the boss section and the mass section facing each other through the

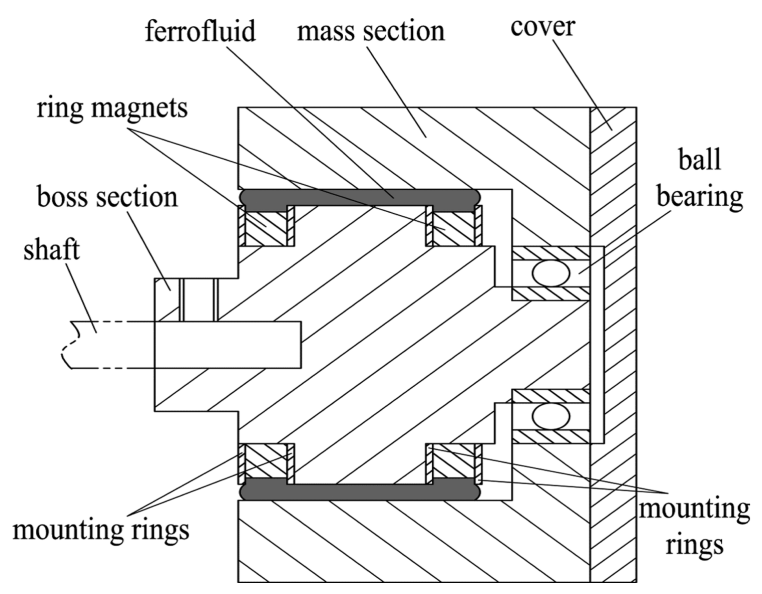

Fig. 10. A ferrofluid damper device for a motor.

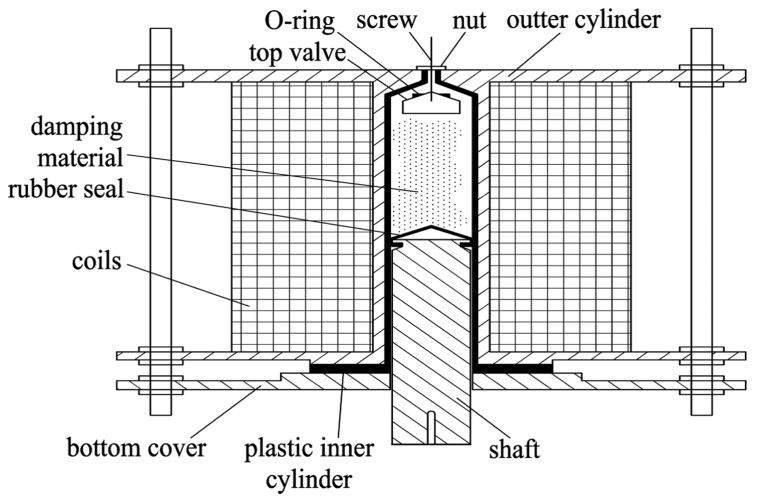

Fig. 11. A smart colloidal damper with ferrofluid.

gap. When the external temperature changes, the viscosity of ferrofluid varies, thus the damping effect of the damper device is affected. While this phenomenon could be dispelled by a change in gap dimension caused by the difference in expansion.

In summary, performance benefits of ferrofluid dampers applied in motor, especially in stepper motor include: increased positional accuracy, reduced settling time, and reduced torsional oscillations. Moreover, they are easily attached to the motor shaft, and have no maintenance requirements. They also can be applied in X-Y-Z plotters, printers, optical scanners, robotics, milling machines, and so on.

Those ferrofluid dampers introduced earlier are all passive ones. Considering the nanoflow damping mechanism and the magnetic properties of ferrofluid, Zhou and Sun [33] developed a smart colloidal damper with ferrofluid, actually a semi-active damper, and the structure is illustrated in Fig. 11. The damping material consists of water-based ferrofluid doped with porous micro-particles. The material of the shaft is copper, and the plastic inner cylinder is used for reducing the oil seal friction. Inside the aluminum and plastic cylinders, a uniform magnetic field is produced by the copper coils. This smart colloidal damper with ferrofluid is proved to have extraordinary performances compared to other smart dampers: simplicity, low heat generation, on demand controllability, and large stoke. In addition, it could avoid the drawback of self-aggregation of polarized in ER/MR dampers. It is suitable for developing advanced semi-active vibration control systems. And the brief comparison for ferrofluid dampers is shown in Table 1.

\subsection{Ferrofluid Vibration Isolators}

As one of the most powerful tools to control vibration, vibration isolation is a procedure by which the undesirable effects of vibration are reduced [34]. Basically, it 
Table 1. Comparison of ferrofluid dampers.

\begin{tabular}{ccccc}
\hline \hline Reference & Year & Type & Applications & Carrier of ferrofluid \\
\hline NASA [9] & 1967 & Passive & RAE Satellite & Alpha methyl naphthalene \\
Leo et al. [10] & 1970 & passive & Dynamic system & $\mathrm{n} / \mathrm{r}$ \\
Moskowitz et al. [30] & 1978 & Passive & Stepper motors and similar devices & $\mathrm{n} / \mathrm{r}$ \\
Miller et al. [31] & 1980 & passive & Rotating machinery & $\mathrm{n} / \mathrm{r}$ \\
Kogure et al. [32] & 1992 & passive & Motor & $\mathrm{n} / \mathrm{r}$ \\
Calarasu et al. [29] & 1999 & Passive & $\mathrm{n} / \mathrm{r}$ & Kerosene \\
Zhou et al. [33] & 2008 & Semi-active & Advanced semi-active vibration control systems & Water \\
\hline
\end{tabular}

$\mathrm{n} / \mathrm{r}$ : not report

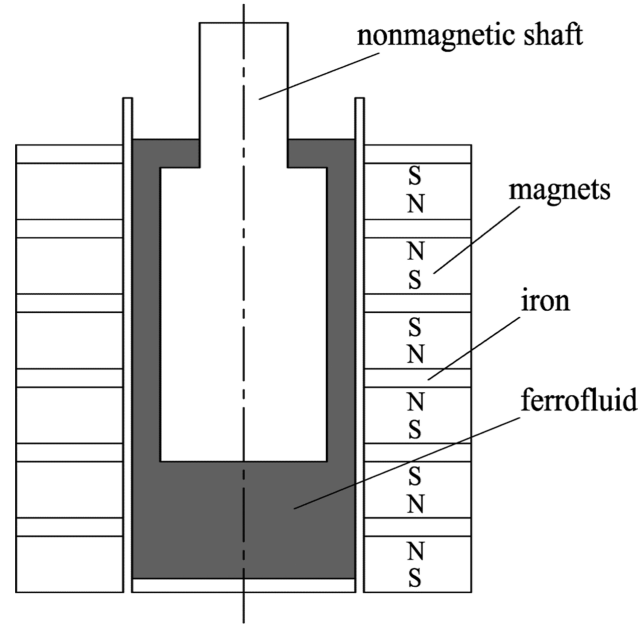

Fig. 12. A vibration isolator using ferrofluid.

involves the insertion of a resilient member (or isolator) between the vibrating mass (or equipment or payload) and the source of vibration so that a reduction in the dynamic response of the system is achieved under specified conditions of vibration excitation. An isolation system is deemed to be active or passive depending on whether or not external power is required for the isolator to perform its function. A passive isolator consists of a resilient member and energy dissipater, while an active one involves sensors and actuators that produce destructive interference to cancel out incoming vibration [35].

In order to damp the low frequency resonant $(2-3 \mathrm{~Hz})$ vibration of an isolating table, scholars from Japan [36] proposed a vibration isolator using ferrofluid (see Fig. 12). By utilizing the levitation force acting on a nonmagnetic material in ferrofluid under a nonuniform magnetic field, a piston type damper with no solid contact is achieved, which can generate a strong damping drag force due to the increase of an apparent viscosity of ferrofluid by applying a magnetic field. The vibration isolating table equipped with the ferrofluid vibration isolator can avoid the adverse effects from the ground noise effectively through inhibiting the resonance vibration, which can be

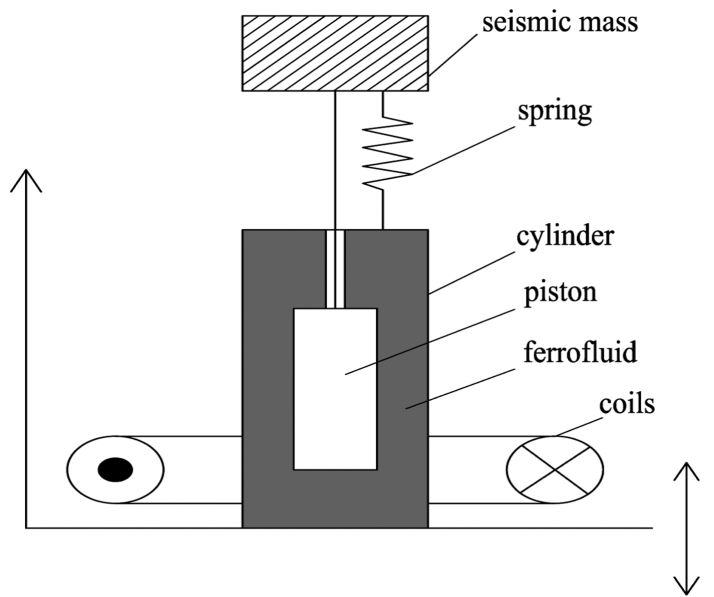

Fig. 13. A ferrofluid active vibration isolator.

used for precision instruments such as a balance, a precise processing machine or an optical tool. Exactly, it is applicable only to mitigate the vertical component of vibrational noises, while an isolation of linear or rotational component of vibrational noises in horizontal plane needs further consideration.

The foregoing structure in Fig. 12 is passive, while Fukuda et al. [37] presented a ferrofluid active vibration isolator which was used to control the vibration of a spring-mass system (see Fig. 13). The object levitated in ferrofluid is also nonmagnetic, and a controllable magnetic field is imposed by a couple of coils, while a constant magnetic field is generated by permanent magnets in the previous configuration. This system is composed of a ferrofluid filled cylinder, a piston submerged in ferrofluid, a seismic mass connected to the piston, and a spring attached between cylinder and mass. Numerical experiments proved the magnetic field necessary for stopping vibration of the mass. The steady vibration could be kept extremely small because the transient vibration is suppressed effectively by the magnetic field generated by a couple of coils. It is worth mentioning that the magnetic field generated by the coils is much weaker than that of permanent magnets, and thus selection of ferrofluid with 


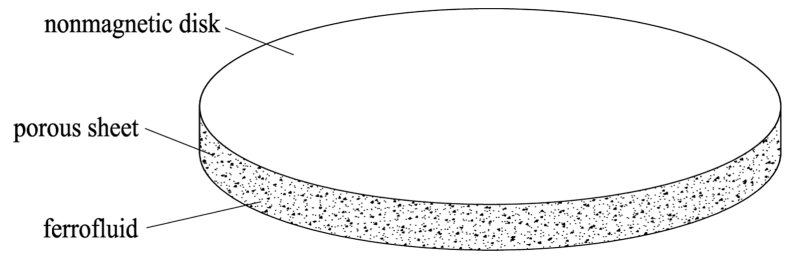

Fig. 14. A porous elastic sheet fluid vibration isolator.

higher magnetization performance may make some sense. Because of the weakness of the magnetic force acting on the ferrofluid, a ferrofluid active vibration isolator was quite difficult to realize at that time, and no experiment was carried out in their investigations.

In the following year, Kamiyama [38], a co-author of the previous article, continued the study on the control of active vibration isolator theoretically and experimentally, with the help of a high quality ferrofluid, namely hydrocarbon-based ferrofluid. By applying a neural network controller, Kamiyama et al. [39] proposed a new controlling method for the ferrofluid active vibration isolator. Meanwhile, a ferrofluid with iron-nitride particles was examined to increase the controlling force. Besides, the structure of a piston immersed in a ferrofluid can be also used as an actuator, and this concept was verified by Olaru et al. [40, 41] from Romania who has done a rather in-depth study till now.

As porous micro-particles were employed in on-demand active damper [33], Liu [42] developed a porous elastic sheet fluid vibration isolator, as depicted in Fig. 14. It comprises two parallel circular disks of nonmagnetic material, between which porous elastic sheets containing ferrofluid are inserted. An analytical estimation in porous elastic sheets efficiency of this isolator was presented, and discussions about effects of damping with a magnetic field and porosity of porous sheets were carried out. The results demonstrated the ferrofluid based porous sheet vibration isolator performed better than one without any porous sheets, and that with porosity of porous sheets decreasing, anti-shock performance is improved. Table 2 enumerates the brief comparison for ferrofluid vibration isolators.

\subsection{Ferrofluid Dynamic Vibration Absorbers}

A dynamic vibration absorber is an auxiliary massspring system which tends to neutralize vibration of a primary system to which it is attached. It is generally composed of mass, spring, and damping elements. It absorbs adverse energy of the primary system by resonance with vibration, and dissipates the energy through its damping element.

To reduce low-frequency oscillations, researchers from Belarus [43] proposed a dynamic vibration absorber based on ferrofluid, with a self-levitating magnet in ferrofluid. Krakov [44] studied the radial flow of ferrofluid under the piston of a ferrofluid dynamic vibration absorber, and has proved that viscous properties of ferrofluid play an important role in the intensity of energy dissipation. Afterwards, Bashtovoi et al. [45-49] conducted further studies on ferrofluid dynamic vibration absorbers, in which the ferrofluid played both the role of support and the damping element.

In 2002, Bashtovoi et al. [46] investigated the dynamics of a ferrofluid dynamic vibration absorber, and the schematic is illustrated in Fig. 15. Owing to external oscillatory inertia forces, the permanent magnet moves inside the body of the absorber, which in turn results in a viscous dissipation of the oscillating system energy. Though the configuration of the dynamic vibration absorber is quite simple, it has been found to have a good performance for suppression of oscillations of small amplitude and low frequency. Furthermore, it owns the advantages of high

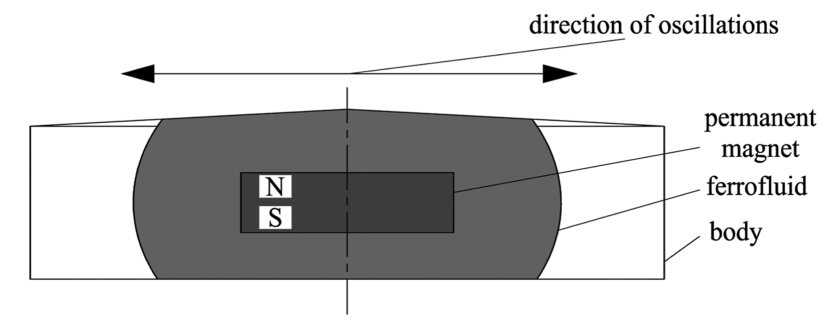

Fig. 15. A ferrofluid dynamic vibration absorber with a selflevitating permanent magnet.

Table 2. Comparison of ferrofluid vibration isolators.

\begin{tabular}{ccccc}
\hline \hline Reference & Year & Type & Applications & Carrier of ferrofluid \\
\hline Nakatsuka et al. [36] & 1987 & Passive & Precise instruments & Dioctylagipate \\
Fukuda et al. [37] & 1998 & Active & Precise machine technology & $\mathrm{n} / \mathrm{r}$ \\
Kamiyama et al. [38] & 1999 & Active & $\mathrm{n} / \mathrm{r}$ & Hydrocarbon \\
Kamiyama et al. [39] & 2002 & Active & Precise machine technology & Kerosene \\
Liu et al. [42] & 2009 & Passive & Small size precision equipments & $\mathrm{n} / \mathrm{r}$ \\
\hline
\end{tabular}

$\mathrm{n} / \mathrm{r}$ : not report 


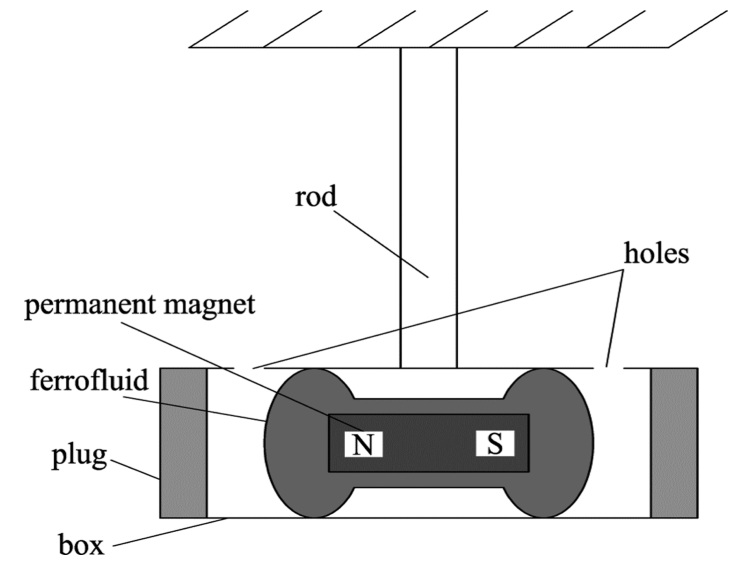

Fig. 16. A ferrofluid dynamic vibration absorber for rod vibrations.

reliability and no energy supply requirement. Microvibration caused by disturbance sources on board space craft could severely degrade the working environment of sensitive payloads [35]. This type of dynamic vibration absorber was expected to be used in spacecraft technology. Subsequently, these researchers investigated the support function of ferrofluid experimentally and numerically [47], and they devoted to the hydrodynamic and dissipation processes in absorber systems with Finite Element Method [48].

Figure 15 exhibits a passive absorber, although Fig. 16 has a similar configuration, Wang et al. [8] from France modified the structure by adding a magnetic interaction force on the seismic mass composed of magnet and ferrofluid. An appropriate selection of the amplitude and initial phase of the interaction force can obtain the optimization motion of the magnet, and then a passive absorber became an active one. The simulation results indicated the possibility of getting an improvement of the damping effect with this active control method.

A new type of ferrofluid dynamic vibration absorber combined a specially designed unit was applied for a patent by Bashtovoi et al. [49] in 2007, as shown in Fig.

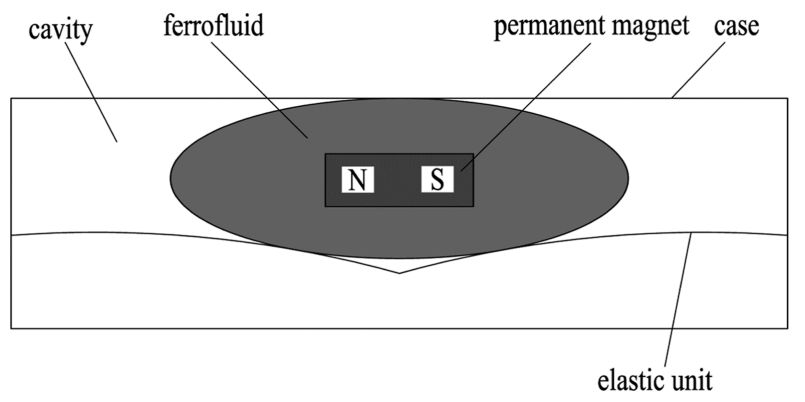

Fig. 17. A ferrofluid dynamic vibration absorber with an elastic unit.



Fig. 18. A ferrofluid dynamic vibration absorber with an annular magnet.

17. The unit is used to stabilize the ferrofluid (with a permanent magnet suspended inside) in a defined position of the cavity, and it could be an elastic unit, a gaseous unit or a rigid wall. By varying pressure of the gas or liquid in another cavity located between a wall of the case and the elastic unit, deformation of the unit is realized. This patent may be employed on a satellite antenna.

Researchers from China [50-52] have shown great interests in ferrofluid dynamic vibration absorbers, and one of their proposed structures is shown in Fig. 18. This structure is constituted of an annular magnet immersed in ferrofluid, which is contained within a cylindrical tube constructed of nonmagnetic material. Ferrofluid acts as support and damping element at the same time. On one hand, based on buoyant levitation of ferrofluid, the magnet is suspended stably. On the other hand, because of the mechanical oscillation, the flow of ferrofluid caused by the motion of the magnet relative to the tube induces the viscous dissipation of the mechanical energy. An elastic beam with a cantilever structure was used to conduct the free oscillations experiments, in order to check the damping capability of the ferrofluid dynamic vibration absorber. Dependence of the logarithmic decay rate on parameters of the absorber was investigated, taking the radius of the magnet for example.

A novel type of damping device, which absorbs energy of structural vibration by tuning the frequency of sloshing fluid within a container to the structural frequency was developed by researchers, namely Tuned Liquid Damper (TLD). To improve the performance of TLD, Abe et al. [53] employed ferrofluid as the working fluid in 1998.

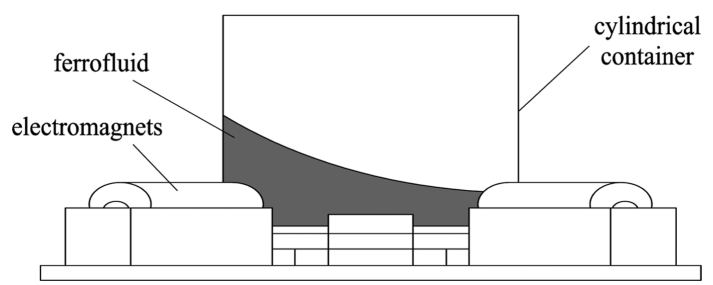

Fig. 19. An active tuned liquid damper. 


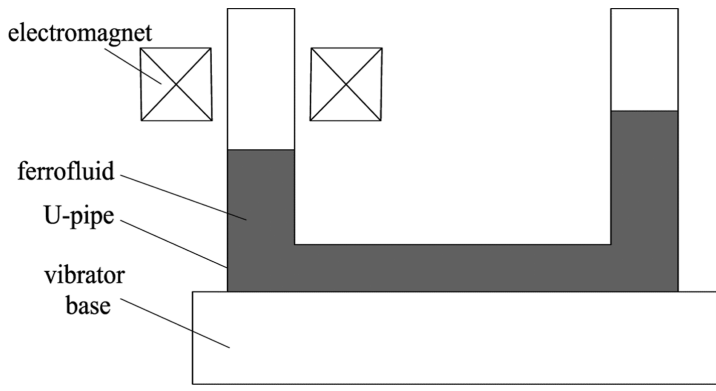

Fig. 20. A tuned liquid column damper using ferrofluid.

Further studies were conducted by Sawada et al. [54-58] from Japan. Figure 19 illustrates an active absorber, and it was actually called an active tuned liquid damper in which ferrofluid activated by electromagnets was used to improve its performance. It was ascertained that this active TLD, compared to conventional devices, had better performances in terms of vibration suppression and lower sensitivity to tuning error. Sawada et al. conducted a series of experiments, and at the same time, analytical models and numerical simulations were developed.

Similar to the concept of previous TLD, Tuned Liquid Column Damper (TLCD) utilizes a column-like container to absorb the energy via the motion of the liquid mass. Researchers from Japan [59-64] attempted a new TLCD using a ferrofluid as the working fluid, which could be categorized as a dynamic vibration absorber, as shown in Fig. 20. It is also known as Magnetic Fluid TLCD (MFTLCD). And it was a semi-active one, of which the natural frequency could be changed via a magnetic field. This ferrofluid dynamic vibration absorber was expected to be used as an effective vibration suppression mechanism for wind excitations and earthquake-induced vibrations in building structures. Table 3 summarizes the ferrofluid dynamic vibration absorbers demonstrated to date together with their research focus and applications.

\subsection{Vibration Energy Harvesters Based on Ferrofluid}

Recently, Alazemi et al. [65, 66] proposed a novel Tuned Magnetic Fluid Damper (TMFD) which was capable of mitigating structural vibrations and harvesting vibration energy simultaneously. The energy harvesting TMFD mounted on a vibrating structure is constituted of a rectangular container which carries a magnetized ferrofluid. However, the concept of vibration energy harvester based on ferrofluid was proposed by Bibo et al. [67] in 2012, as shown in Fig. 21. It was an electromagnetic micro-power generator, which transformed the sloshing motions of a ferrofluid column into electricity. The feasi-

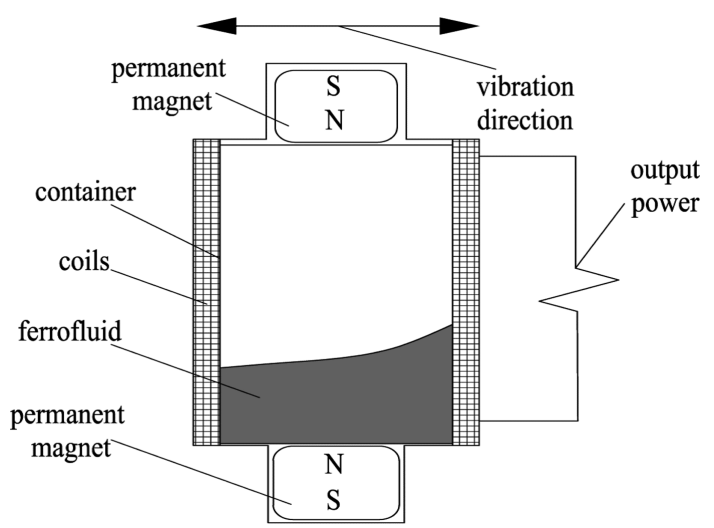

Fig. 21. A ferrofluid-based vibration energy harvester.

Table 3. Comparison of ferrofluid dynamic vibration absorbers.

\begin{tabular}{|c|c|c|c|c|}
\hline Reference & Year & Type & Research Focus & Applications \\
\hline Krakov [44] & 1999 & Passive & Energy dissipation & Reduction of low-frequency oscillations \\
\hline Bashtovoi et al. [46] & 2002 & Passive & Energy dissipation & $\begin{array}{l}\text { Spacecraft Technology, solar panels, } \\
\text { satellite antenna }\end{array}$ \\
\hline Bashtovoi et al. [49] & 2007 & Passive & Several new structures & The same as above \\
\hline Wang et al. [8] & 2003 & Active & Efficiency of damping system and active control & Rod vibration damping systems \\
\hline Yang et al. $[50,52]$ & 2013,2015 & Passive & $\begin{array}{l}\text { Hydrodynamics and energy dissipation, } \\
\text { magnetic levitation force }\end{array}$ & $\mathrm{n} / \mathrm{r}$ \\
\hline Yao et al. [51] & 2015 & Passive & Energy dissipation and magnetic restoring force & Spacecraft technology \\
\hline Abe et al. [53] & 1998 & Active & $\begin{array}{l}\text { Characteristics of sloshing motion } \\
\text { of ferrofluid subject to dynamic magnetic field }\end{array}$ & $\begin{array}{l}\text { Reduction of wind excitations and } \\
\text { earthquake-induced vibrations in } \\
\text { building structures }\end{array}$ \\
\hline Sawada et al. [54-58] & 2001 2011 & Active & $\begin{array}{l}\text { A series of studies on characteristics of tuned } \\
\text { fluid damper using ferrofluid }\end{array}$ & The same as above \\
\hline Sawada et al. [59-64] & 2002 2016 & Semi-active & $\begin{array}{l}\text { A series of studies on characteristics of tuned } \\
\text { liquid column damper using ferrofluid }\end{array}$ & The same as above \\
\hline
\end{tabular}

$\mathrm{n} / \mathrm{r}$ : not report 
Table 4. Comparison of ferrofluid vibration energy harvesters.

\begin{tabular}{|c|c|c|c|c|}
\hline Reference & Year & Carrier of Ferrofluid & Maximum Output Voltage & Maximum Output Power \\
\hline Bibo et al. [67] & 2012 & hydrocarbon & $18 \mathrm{mV}$ & $1 \mu \mathrm{W}$ \\
\hline Chae et al. [68] & 2013 & $\mathrm{n} / \mathrm{r}$ & $0.47 \mathrm{~V}$ & $71.26 \mu \mathrm{W}$ \\
\hline Alazemi et al. [65] & 2013 & hydrocarbon & $8 \mathrm{mV}^{\mathrm{a}}$ & $0.6 \mathrm{~mW} / \mathrm{g}$ \\
\hline Oh et al. [69] & 2014 & hydrocarbon-oil & $\mathrm{n} / \mathrm{r}$ & $0.25 \mu \mathrm{W}$ \\
\hline Wang et al. [70] & 2015 & kerosene & $\mathrm{n} / \mathrm{r}$ & $0.27 \mathrm{~mW}$ \\
\hline Wang et al. [71] & 2015 & kerosene & $\mathrm{n} / \mathrm{r}$ & $0.26 \mathrm{~mW}$ \\
\hline Wang et al. [72] & 2015 & $\mathrm{n} / \mathrm{r}$ & $0.58 \mathrm{mV}$ & $36 \mathrm{nW}$ \\
\hline Monroe et al. [73] & 2015 & Water & $20 \mu \mathrm{V}^{\mathrm{a}}$ & $\mathrm{n} / \mathrm{r}$ \\
\hline Alazemi et al. [74] & 2015 & $\mathrm{n} / \mathrm{r}$ & $\mathrm{n} / \mathrm{r}$ & $80 \mathrm{~mW} / \mathrm{g}$ \\
\hline Kim et al. [75] & 2015 & water & $0.1 \mathrm{~V}^{\mathrm{a}}$ & $\mathrm{n} / \mathrm{r}$ \\
\hline Kim et al. [76] & 2015 & oil & $\mathrm{n} / \mathrm{r}$ & $19.3 \mu \mathrm{W}$ \\
\hline Kim [77] & 2015 & hydrocarbon-oil & $8 \mathrm{mV}^{\mathrm{a}}$ & $\mathrm{n} / \mathrm{r}$ \\
\hline
\end{tabular}

$\mathrm{n} / \mathrm{r}$ : not report.

a: estimated or read from figures in reference.

bility and efficiency of the proposed energy harvesting device were studied through experiments. In comparison with traditional electromagnetic generators with solid magnets, the response to extremely small acceleration and feasibility in different shapes provide a potential opportunity to design scalable energy harvesters. From then on, more and more scholars [68-77] have made an effort to research of vibration energy harvesters based on ferrofluid which are briefly summarized in Table 4 .

\section{A Novel Ferrofluid Dynamic Vibration Absorber}

A novel dynamic vibration absorber based on ferrofluid was proposed by the authors of this paper recently, and the schematic is shown in Fig. 22. An annular magnet is sheathed on the outer side of the housing, which is positioned in axial direction by grommet and base. Two cylindrical magnets are fixed on the inner surface of the cover and the inner bottom surface of the housing, respectively. The seismic mass made of copper is placed in the housing, which is levitated by the ferrofluid based on

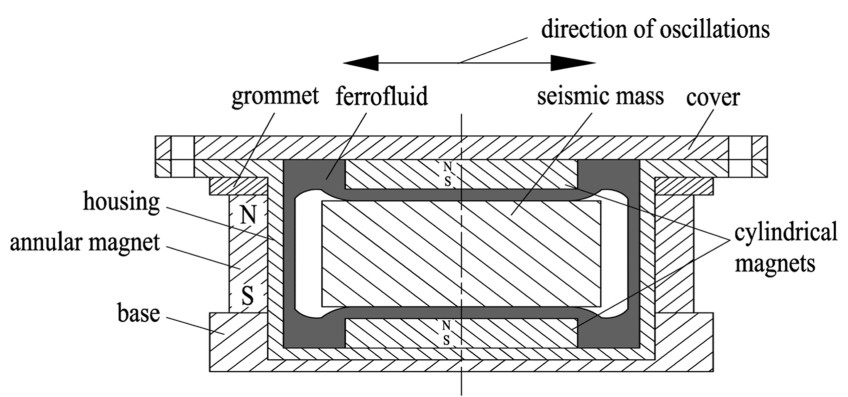

Fig. 22. A novel ferrofluid dynamic vibration absorber.

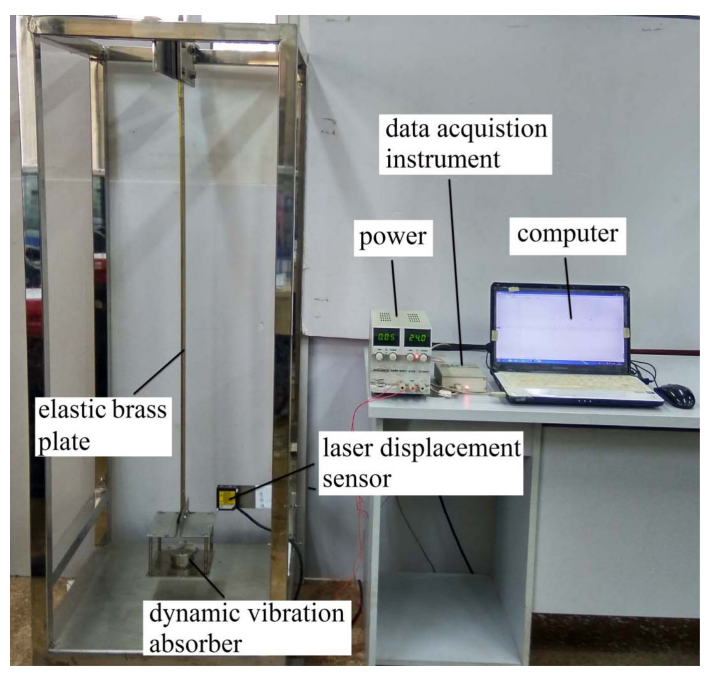

Fig. 23. (Color online) A photograph of the experimental devices.

buoyant levitation of the first kind. When the external oscillation occurs, the seismic mass moves in the chamber, accompanied by the generation of the viscous shearing in ferrofluid, leading to energy dissipation in the system. It is noted that, the ferrofluid does not need to fill the chamber fully for the flexible movement of the seismic mass. Figure 23 shows the experimental apparatus for the proposed dynamic vibration absorber, and plate oscillations with and without dynamic vibration absorber are shown in Fig. 24, separately. Experimental results indicate that the oscillating time could be reduced by about $85 \%$ when the mass of the ferrofluid is $30 \mathrm{~g}$. More and deeper research will be conducted in the future, and this dynamic vibration absorber is expected to be applied in spacecraft technology. 

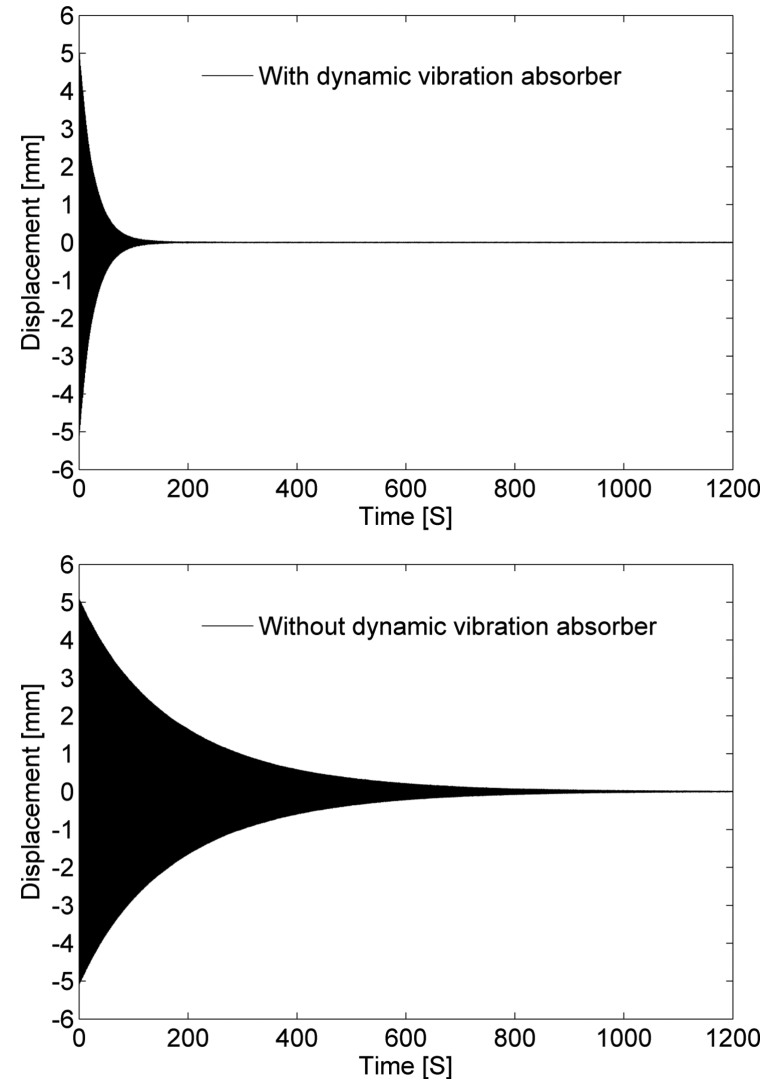

Fig. 24. Diagrams of the plate oscillations with and without the dynamic vibration absorber.

\section{Conclusion}

This study has provided a comprehensive review of the main developments in the field of damping applications of ferrofluids over half a century. The applications are classified into three types, namely dampers, vibration isolators and dynamic vibration absorbers. In addition, vibration energy harvesters based on ferrofluid are simply introduced, and a novel dynamic vibration absorber is developed and evaluated. Among these applications, some of which have been used in practice, many of them still remain in the laboratory research stage.

Clearly, research and development into damping applications of ferrofluids will continue to be a promising and active field. Perspectives of ferrofluid damping devices could be:

1. The investigation on the active damping device with ferrofluid is of great importance, and high quality ferrofluid and better performance of the electromagnet are required. More effective attenuation of oscillations in a wider frequency range will be obtained, by more precise control of the magnetic field.

2. Since ferrofluid has the unique property of being controlled by a magnetic field, damping device in the aerospace may be one of the prospective applications, with more stringent requirements of smaller volume, less weight and higher reliability.

3. Leakage of magnetic flux should not be ignored, because it would affect other peripheral equipment adversely. When the mobile mass is made of magnet, magnetic shielding outside the damper cannot be imposed. Levitation of a nonmagnetic mass in ferrofluid may provide a new way, but there still exist some problems to be considered, such as the stable suspension of nonmagnetic mass.

4. Though the achievable power that can be produced is far less than the requirements currently, vibration energy harvester based on ferrofluid is a highly significant research direction in the future.

It is earnestly hoped that this work would not only provide a valuable reference for further exploring in this field, but also serve as an inspiration for beginners or those preparing to enter this field.

\section{Acknowledgement}

This work was supported by the National Science Foundation of China (grant number 51375039), Creative Groups Development Program of the Ministry of Education of China (grant number IRT13046), and Key Project of Science and Technology Research and Development Program of China Railway Corporation (grant number M15D00190).

\section{References}

[1] S. Odenbach, Lect. Notes. Phys. 594 (2002).

[2] R. E. Rosensweig, Annu. Rev. Fluid. Mech. 19, 437 (1987).

[3] P. Stephen Solomon, Low viscosity magnetic fluid obtained by the colloidal suspension of magnetic particles, U.S. Patent (3215572), (1965).

[4] J. Rabinow, Trans. Am. Inst. Electr. Eng. 67, 1308 (1948).

[5] S. Genc and B. Derin, Curr. Opin. Chem. Eng. 3, 118 (2014).

[6] P. P. Phule and J. M. Ginder, MRS Bull. 23, 19 (1998).

[7] F. Imaduddin, S. A. Mazlan, and H. Zamzuri, Mater. Des. 51, 575 (2013).

[8] Z. Wang, G. Bossis, O. Volkova, V. Bashtovoi, and M. Krakov, J. Intell. Mater. Syst. Struct. 14, 93 (2003).

[9] Feasibility study and model development for a ferrofluid viscous damper, Goddard Space Flight Center, Maryland (1967).

[10] B. Leo and L. Rudolph, Viscous damper using magnetic 
ferrofluid, U.S. Patent (3538469), (1970).

[11] R. Moskowitz, IEEE Spectr. 12, 53 (1975).

[12] K. Raj and R. Moskowitz, J. Magn. Magn. Mater. 85, 233 (1990).

[13] K. Raj, B. Moskowitz, and R. Casciari, J. Magn. Magn. Mater. 149, 174 (1995).

[14] B. D. Moscowitz and K. Raj, Mach. Des. 67, 57 (1995).

[15] J. Popplewell and S. Charles, IEEE Trans. Magn. 17, 2923 (1981).

[16] R. E. Rosensweig, Sci. Am. 247, 136 (1982).

[17] C. Scherer and A. M. Figueiredo Neto, Braz. J. Phys. 35, 718 (2005)

[18] D. Mayer, Adv. Electr. Electron. Eng. 7, 9 (2008).

[19] C. Buzduga, The Third International Symposium on Electrical Engineering and Energy Converters, 24 (2009).

[20] F. Ezekiel, Mechanical Engineering 97, 94 (1975).

[21] K. Raj and R. Moskowitz, IEEE Trans. Magn. 16, 358 (1980).

[22] I. Torres-Díaz and C. Rinaldi, Soft Matter 10, 8584 (2014).

[23] J. Philip and J. M. Laskar, Journal of Nanofluids 1, 3 (2012).

[24] H. Shokrollahi, Mater. Sci. Eng. C 33, 2476 (2013).

[25] R. J. Yang, H. H. Hou, Y. N. Wang, and L. M. Fu, Sens. Actuators: B 224, 1 (2016).

[26] R. E. Rosensweig, Ferrohydrodynamics, Cambrige University Press, Cambridge (1985).

[27] R. E. Rosensweig, AIAA J. 4, 1751 (1966).

[28] R. E. Rosensweig, Nature 210, 613 (1966).

[29] D. Calarasu, C. Cotae, and R. Olaru, J. Magn. Magn. Mater. 201, 401 (1999).

[30] R. Moskowitz, P. Stahl, and W. R. Reed, Inertia damper using ferrofluid, U.S. Patent (4123675), (1978).

[31] D. L. Miller, Magnetic viscous damper, U.S. Patent (4200003), (1980).

[32] T. Kogure, Damper device for a motor, U.S. Patent (5081882), (1992).

[33] G. Zhou and L. Sun, Smart Mater. Struct. 17, 055023 (2008).

[34] S. S. Rao and F. F. Yap, Mechanical vibrations (forth edition), Addison-Wesley, New York (1995).

[35] C. Liu, X. Jing, S. Daley, and F. Li, Mech. Syst. Signal Process. 56, 55 (2015).

[36] K. Nakatsuka, H. Yokoyama, J. Shimoiizaka, and T. Funaki, J. Magn. Magn. Mater. 65, 359 (1987).

[37] H. Fukuda, K. Ueno, S. Kamiyama, and T. Oyama, JSME Int. J. Ser. B 41, 822 (1998).

[38] S. Kamiyama, Int. J. Mod. Phys. B 13, 2213 (1999).

[39] S. Kamiyama, K. Okamoto, and T. Oyama, Energy Convers. Manage. 43, 281 (2002).

[40] R. Olaru, A. Salceanu, D. Calarasu, and C. Cotae, Sens. Actuators: A 81, 290 (2000).

[41] C. Petrescu and R. Olaru, IEEE International Symposium on Advanced Topics in Electrical Engineering, 374 (2015).

[42] J. Liu, J. Tribology 131, 021801 (2009).

[43] B. M. Berkovsky and V. Bashtovoy, Magnetic fluids and applications handbook, Begell house, New York (1996).

[44] M. S. Krakov, J. Magn. Magn. Mater. 201, 368 (1999).

[45] V. Bashtovoi, D. Kabachnikov, A. Reks, L. Suloeva, G. Bossis, and O. Volkova, Magnetohydrodynamics 36, 190 (2000).

[46] V. G. Bashtovoi, D. N. Kabachnikov, A. Y. Kolobov, V. B. Samoylov, and A. V. Vikoulenkov, J. Magn. Magn. Mater. 252, 312 (2002).

[47] V. G. Bashtovoi, G. Bossis, D. N. Kabachnikov, M. S. Krakov, and O. Volkova, J. Magn. Magn. Mater. 252, 315 (2002).

[48] V. Bashtovoi, O. Lavrova, T. Mitkova, V. Polevikov, and L. Tobiska, J. Magn. Magn. Mater. 289, 207 (2005).

[49] V. Bashtovoi, A. Reks, P. Kuzhir, G. Bossis, A. Vikulenkov, A. Moisheev, and N. Markachev, Inertial damper for E.g. satellite antenna, has case with cavity comprising complex including magnetic field source introduced In magnetic fluid, and elastic unit permitting to stabilize complex in defined position of cavity, F.R. Patent (2894004 A1), (2007).

[50] W. Yang, D. Li, and Z. Feng, J. Vib. Control 19, 183 (2013).

[51] J. Yao, J. Chang, D. Li, and X. Yang, J. Magn. Magn. Mater. 402, 28 (2015).

[52] W. Yang, J. Vib. Control (2015).

[53] M. Abe, Y. Fujino, and S. Kimura, 5th Annual International Symposium on Smart Structures and Materials, 620 (1998).

[54] Y. Ohira, H. Houda, and T. Sawada, Int. J. Appl. Electromagn. Mech. 13, 71 (2001).

[55] S. Horie, M. Shimoda, K. Ohno, J. Nakamura, and T. Sawada, Int. J. Appl. Electromagn. Mech. 25, 139 (2007).

[56] K. Ohno, M. Shimoda, and T. Sawada, J. Phys.: Condens. Matter 20, 204146 (2008).

[57] K. Ohno and T. Sawada, Int. J. Appl. Electromagn. Mech. 33, 1411 (2010).

[58] K. Ohno, H. Suzuki, and T. Sawada, J. Magn. Magn. Mater. 323, 1389 (2011).

[59] M. Ohaba, Y. Suzuki, T. Sawada, Y. Z. Liu, M. Takeuchi, and T. Tanahashi, J. Magn. Magn. Mater. 252, 306 (2002).

[60] H. Masuda, T. Oyamada, K. Ikari, and T. Sawada, J. Jpn. Soc. Appl. Electromagn. Mech. 21, 228 (2013).

[61] T. Oyamada, H. Masuda, K. Ikari, and T. Sawada, Magnetohydrodynamics 49, 515 (2013).

[62] T. Oyamada, H. Masuda, K. Ikari, and T. Sawada, Int. J. Appl. Electromagn. Mech. 45, 659 (2014).

[63] K. Ikari, H. Masuda, T. Oyamada, and T. Sawada, Materials Science Forum 792, 275 (2014).

[64] S. Kondo, K. Ikari, and T. Sawada, Materials Science Forum 856, 21 (2016).

[65] S. F. Alazemi, M. F. Daqaq, S. F. Alazemi, and M. F. Daqaq, ASME 2013 Conference on Smart Materials, Adaptive Structures and Intelligent Systems, V002T07A029 (2013)

[66] S. F. Alazemi, A. Bibo, and M. F. Daqaq, ASME 2014 
Conference on Smart Materials, Adaptive Structures and Intelligent Systems, V002T07A008 (2014).

[67] A. Bibo, R. Masana, A. King, G. Li, and M. F. Daqaq, Phys. Lett. A 376, 2163 (2012).

[68] S. H. Chae, S. Ju, Y. Choi, S. Jun, S. M. Park, S. Lee, H. W. Lee, and C. Ji, Journal of Physics: Conference Series, 914 (2013).

[69] D. W. Oh, D. Y. Sohn, D. G. Byun, and Y. S. Kim, IEEE International Conference on Electrical Machines and Systems, 2033 (2014).

[70] S. Wang and D. Li, J. Korean Phys. Soc. 67, 818 (2015).

[71] S. Wang and D. Li, Electron. Lett. 51, 1693 (2015).
[72] Y. Wang, Q. Zhang, L. Zhao, and E. S. Kim, IEEE International Conference on MICRO Electro Mechanical Systems, 122 (2015).

[73] J. G. Monroe and S. M. Thompson, Proc. SPIE 9493, 94930G (2015).

[74] S. F. Alazemi, A. Bibo, and M. F. Daqaq, Eur. Phys. J. Spec. Top. 224, 2993 (2015).

[75] D. Kim and K. Yun, Journal of Physics: Conference Series 660, 012108 (2015).

[76] D. Kim, S. Yu, B. G. Kang, and K. S. Yun, J. Microelectromech. S. 24, 516 (2015).

[77] Y. S. Kim, J. Magn. 20, 252 (2015). 